\title{
Review of: "Can magnitude and days with fever predict urinary tract infection in infants with fever without source?"
}

Roberto Velasco

Potential competing interests: The author(s) declared that no potential competing interests exist.

This manuscript is a retrospective study that tries to determine the prevalence of urinary infection (UTI) in infants under 36 months with fever without a source, as well as the existence of risk factors that increase the probability of this diagnosis, and allow the selection of a group of patients in whom the tests have a greater diagnostic value.

The study is relevant, and although it is well structured, there are some typographical errors that make it difficult to read. (For example, in the method it is indicated that a temperature above $38.8 \circ \mathrm{C}$ was considered fever, but in Table $170 \%$ of patients with a temperature $<39{ }^{\circ} \mathrm{C}$ appear, so I understand that fever was considered at a temperature $>38.0$ C. Also, in Table 1 there is some sign " $<$ " and ">" misspelled, on days of fever, for example). It is advisable to review these errors, since they diminish the value of the manuscript.

Regarding the study method, the fact that it is retrospective necessarily reduces the validity of the results. Although it is true that the number of patients treated per year in the hospital is not indicated, the low number of patients recruited in three years is striking, taking into account that they are patients under 36 months of age. Likewise, the prevalence described in many series for this age group is less than $10 \%$, with a prevalence of $20 \%$ more typical among infants under three months of age, which here is less than $7 \%$, but the prevalence of UTI is $>70 \%$. All this, in addition to the high percentage of patients with underlying pathology or alteration of the general state, suggest that the population that underwent a urinalysis was more selected, making the prevalence value obtained less reliable.

Another problem that may increase the prevalence of UTI is the fact that only urine culture has been considered as a diagnostic criterion for UTI. The recommendations of other clinical guidelines, such as those of the AAP, advise considering as true UTIs those that, in addition to a urine culture with growth of pathogenic bacteria, also present an altered urine analysis. It would be interesting if the authors reevaluated their analysis in these patients, albeit as a secondary analysis, and compared the results.

In Table 2 of the results, it is more informative for the reader if the percentages indicated were those of the 
row, not those of the column.

In Table 2 there are 41 patients with a temperature $<38.0^{\circ} \mathrm{C}$. According to the inclusion criteria of the study, these patients should have been excluded, since they are not patients with fever. Please explain if it is a typo, or else I recommend redoing the calculations without these patients. 